\title{
Bounded Entanglement Entropy in the Quantum Ising Model
}

\author{
Geoffrey R. Grimmett ${ }^{1}$ (D) Tobias J. Osborne ${ }^{2}$. Petra F. Scudo ${ }^{3}$
}

Received: 28 June 2019 / Accepted: 8 November 2019 / Published online: 2 December 2019

(c) The Author(s) 2019

\begin{abstract}
A rigorous proof is presented of the boundedness of the entanglement entropy of a block of spins for the ground state of the one-dimensional quantum Ising model with sufficiently strong transverse field. This is proved by a refinement of the stochastic geometric arguments in the earlier work by Grimmett et al. (J Stat Phys 131:305-339, 2008). The proof utilises a transformation to a model of classical probability called the continuum random-cluster model. Our method of proof is fairly robust, and applies also to certain disordered systems.
\end{abstract}

Keywords Quantum Ising model · Entanglement · Entropy · Area law · Random-cluster model

Mathematics Subject Classification $82 \mathrm{~B} 20 \cdot 60 \mathrm{~K} 35$

\section{The Quantum Ising Model and Entanglement}

The purpose of this note is to give a rigorous proof of the area law for entanglement entropy in the quantum Ising model in one dimension. This is achieved by an elaboration of the stochastic geometrical approach of [21]. We prove the boundedness of entanglement entropy of a block of spins of size $L+1$ in the ground state of the model with sufficiently strong transverse field, uniformly in $L$. The current paper is presented as a development of the earlier

Communicated by Irene Giardina.

Geoffrey R. Grimmett

grg@statslab.cam.ac.uk

Tobias J. Osborne

tobias.j.osborne@gmail.com

Petra F. Scudo

pscudo@gmail.com

1 Centre for Mathematical Sciences, University of Cambridge, Wilberforce Road, Cambridge CB3 OWB, UK

2 Institut für Theoretische Physik, Leibniz Universität Hannover, Appelstr. 2, 30167 Hannover, Germany

3 European Commission, Joint Research Centre, Directorate B, Growth \& Innovation Unit B6, Digital Economy Via E. Fermi, 2749, 21027 Ispra, VA, Italy 
work [21] by the same authors, to which the reader is referred for details of the background and basic theory.

The quantum Ising model in question is defined as follows. We consider a block of $L+1$ spins in a line of length $2 m+L+1$. Let $L \geq 0$. For $m \geq 0$, let

$$
\Delta_{m}=\{-m,-m+1, \ldots, m+L\}
$$

be a subset of the one-dimensional lattice $\mathbb{Z}$, and attach to each vertex $x \in \Delta_{m}$ a quantum spin- $\frac{1}{2}$ with local Hilbert space $\mathbb{C}^{2}$. The Hilbert space $\mathcal{H}$ for the system is $\mathcal{H}=\bigotimes_{x=-m}^{m+L} \mathbb{C}^{2}$. A convenient basis for each spin is provided by the two eigenstates $|+\rangle=\left(\begin{array}{l}1 \\ 0\end{array}\right),|-\rangle=\left(\begin{array}{l}0 \\ 1\end{array}\right)$, of the Pauli operator

$$
\sigma_{x}^{(3)}=\left(\begin{array}{ll}
1 & 0 \\
0 & -1
\end{array}\right)
$$

at the site $x$, corresponding to the eigenvalues \pm 1 . The other two Pauli operators with respect to this basis are represented by the matrices

$$
\sigma_{x}^{(1)}=\left(\begin{array}{ll}
0 & 1 \\
1 & 0
\end{array}\right), \quad \sigma_{x}^{(2)}=\left(\begin{array}{ll}
0 & -i \\
i & 0
\end{array}\right) .
$$

A complete basis for $\mathcal{H}$ is given by the tensor products (over $x$ ) of the eigenstates of $\sigma_{x}^{(3)}$. In the following, $|\phi\rangle$ denotes a vector and $\langle\phi|$ its adjoint. As a notational convenience, we shall represent sub-intervals of $\mathbb{Z}$ as real intervals, writing for example $\Delta_{m}=[-m, m+L]$.

The spins in $\Delta_{m}$ interact via the quantum Ising Hamiltonian

$$
H_{m}=-\frac{1}{2} \sum_{\langle x, y\rangle} \lambda \sigma_{x}^{(3)} \sigma_{y}^{(3)}-\sum_{x} \delta \sigma_{x}^{(1)},
$$

generating the operator $e^{-\beta H_{m}}$ where $\beta$ denotes inverse temperature. Here, $\lambda \geq 0$ and $\delta \geq 0$ are the spin-coupling and external-field intensities, respectively, and $\sum_{\langle x, y\rangle}$ denotes the sum over all (distinct) unordered pairs of neighbouring spins. While we phrase our results for the translation-invariant case, our approach can be extended to disordered systems with couplings and field intensities that vary across $\mathbb{Z}$, much as in [21, Sect. 8]. See Theorem 1.5.

The Hamiltonian $H_{m}$ has a unique pure ground state $\left|\psi_{m}\right\rangle$ defined at zero temperature (as $\beta \rightarrow \infty$ ) as the eigenvector corresponding to the lowest eigenvalue of $H_{m}$. This ground state $\left|\psi_{m}\right\rangle$ depends only on the ratio $\theta=\lambda / \delta$. We work here with a free boundary condition on $\Delta_{m}$, but we note that the same methods are valid with a periodic (or wired) boundary condition, in which $\Delta_{m}$ is embedded on a circle.

Write $\rho_{m}(\beta)=e^{-\beta H_{m}} / \operatorname{tr}\left(e^{-\beta H_{m}}\right)$, and

$$
\rho_{m}=\lim _{\beta \rightarrow \infty} \rho_{m}(\beta)=\left|\psi_{m}\right\rangle\left\langle\psi_{m}\right|
$$

for the density operator corresponding to the ground state of the system. The ground-state entanglement of $\left|\psi_{m}\right\rangle$ is quantified by partitioning the spin chain $\Delta_{m}$ into two disjoint sets $[0, L]$ and $\Delta_{m} \backslash[0, L]$ and by considering the entropy of the reduced density operator

$$
\rho_{m}^{L}=\operatorname{tr}_{\Delta_{m} \backslash[0, L]}\left(\left|\psi_{m}\right\rangle\left\langle\psi_{m}\right|\right) .
$$

One may similarly define, for finite $\beta$, the reduced operator $\rho_{m}^{L}(\beta)$. In both cases, the trace is performed over the Hilbert space of spins belonging to $\Delta_{m} \backslash[0, L]$. Note that $\rho_{m}^{L}$ is a positive semi-definite operator on the Hilbert space $\mathcal{H}_{L}$ of dimension $d=2^{L+1}$ of spins indexed by 
the interval $[0, L]$. By the spectral theorem for normal matrices [10], this operator may be diagonalised and has real, non-negative eigenvalues, which we denote in decreasing order by $\lambda_{j}^{\downarrow}\left(\rho_{m}^{L}\right)$.

Definition 1.1 The entanglement (entropy) of the interval $[0, L]$ relative to its complement $\Delta_{m} \backslash[0, L]$ is given by

$$
S\left(\rho_{m}^{L}\right)=-\operatorname{tr}\left(\rho_{m}^{L} \log _{2} \rho_{m}^{L}\right)=-\sum_{j=1}^{2^{L+1}} \lambda_{j}^{\downarrow}\left(\rho_{m}^{L}\right) \log _{2} \lambda_{j}^{\downarrow}\left(\rho_{m}^{L}\right),
$$

where $0 \log _{2} 0$ is interpreted as 0 .

Here are our two main theorems.

Theorem 1.2 Let $\lambda, \delta \in(0, \infty)$ and $\theta=\lambda / \delta$. There exists $C=C(\theta) \in(0, \infty)$, and $a$ constant $\gamma=\gamma(\theta)$ satisfying $0<\gamma<\infty$ if $\theta<2$, such that, for all $L \geq 1$,

$$
\left\|\rho_{m}^{L}-\rho_{n}^{L}\right\| \leq \min \left\{2, C e^{-\gamma m}\right\}, \quad 2 \leq m \leq n .
$$

Furthermore, we may choose such $\gamma$ satisfying $\gamma(\theta) \rightarrow \infty$ as $\theta \downarrow 0$.

Equation (1.5) is in terms of the operator norm:

$$
\left\|\rho_{m}^{L}-\rho_{n}^{L}\right\| \equiv \sup _{\|\psi\|=1}\left|\left\langle\psi\left|\rho_{m}^{L}-\rho_{n}^{L}\right| \psi\right\rangle\right|
$$

where the supremum is taken over all vectors $|\psi\rangle \in \mathcal{H}_{L}$ with unit $L^{2}$-norm.

Remark 1.3 The value $\theta=2$ is critical for the quantum Ising model in one dimension, and therefore the condition $\theta<2$ is sharp for $\gamma>0$ in (1.5). See the discussion following [13, Thm 7.1].

Theorem 1.4 Consider the quantum Ising model (1.2) on $n=2 m+L+1$ spins, with parameters $\lambda, \delta$, and let $\gamma$ be as in Theorem 1.2. If $\gamma>2 \ln 2$, there exists $c_{1}=c_{1}(\theta)<\infty$ such that

$$
S\left(\rho_{m}^{L}\right) \leq c_{1}, \quad m, L \geq 0 .
$$

Weaker versions of Theorems 1.2 and 1.4 were proved in [21, Thms 2.2, 2.8], namely that (1.5) holds subject to a power factor of the form $L^{\alpha}$, and (1.7) holds with $c_{1}$ replaced by $C_{1}+C_{2} \log L$ (and subject to a slightly stronger assumption on $\gamma$ ). As noted in Remark 1.3, Theorem 1.2 is a further strengthening of [21, Thm 2.2] in that (1.5) holds for $\theta<2$, rather then just $\theta<1$. Stronger versions of these two theorems may be proved similarly, with the interactions $\lambda$ and field intensities $\delta$ varying with position while satisfying a suitable condition. A formal statement for the disordered case appears at Theorem 1.5.

There is a considerable and growing literature in the physics journals concerning entanglement entropy in one and more dimensions. For example, paper [17] is an extensive review of area laws. The relationship between entanglement entropy and the spectral gap has been explored in [4,5], and polynomial-time algorithms for simulating the ground state are studied in [6]. Related works include studies of the XY spin chain [1], oscillator systems [7], the XXZ spin chain [8], and free fermions [25]. The connection between correlations and the area-law is explored in [14]. 
We make next some remarks about the proofs of the above two theorems. The basic approach of these mathematically rigorous proofs is via the stochastic geometric representation of Aizenman, Klein, Nachtergaele, and Newman [2,3,23]. Geometric techniques have proved of enormous value in studying both classical systems (including Ising and Potts models, see for example [19]), and quantum systems (see [11-13,15,18,26]).

The proofs of Theorems 1.2, 1.4 and the forthcoming Theorem 1.5 have much in common with those of $[21$, Thms 2.2, 2.8] subject to certain improvements in the probabilistic estimates. The general approach and many details are the same as in the earlier paper, and indeed there is some limited overlap of text. We make frequent reference here to [21], and will highlight where the current proofs differ, while omitting arguments that may be taken directly from [21]. In particular, the reader is referred to [21, Sects. 4, 5] for details of the percolation representation of the ground state, and of the associated continuum random-cluster model. In Sect. 2, we review the relationship between the reduced density operator and the randomcluster model, and we state the fundamental inequalities of Theorem 2.5 and Lemma 2.6. Once the last two results have been proved, Theorems 1.2 and 1.4 follow as in [21]: the first as in the proof of [21, Thm 2.2], and the second as in that of [21, Thm 2.8] (see the notes for the latter included in Sect. 5).

We reflect in Sect. 4 on the extension of our methods and conclusions when the edgecouplings $\lambda$ and field strengths $\delta$ are permitted to vary, either deterministically or randomly, about the line. In this disordered case, the Hamiltonian (1.2) is replaced by

$$
H_{m}=-\frac{1}{2} \sum_{\langle x, y\rangle} \lambda_{x, y} \sigma_{x}^{(3)} \sigma_{y}^{(3)}-\sum_{x} \delta_{x} \sigma_{x}^{(1)}
$$

where the sum is over neighbouring pairs $\langle x, y\rangle$ of $\Delta_{m}$. We write $\lambda=\left(\lambda_{x, x+1}: x \in \mathbb{Z}\right)$ and $\delta=\left(\delta_{x}: x \in \mathbb{Z}\right)$.

Theorem 1.5 Consider the quantum Ising model on $\mathbb{Z}$ with Hamiltonian (1.8), such that, for some $\lambda, \delta>0, \lambda$ and $\delta$ satisfy

$$
\lambda_{x, y} / \delta_{x} \leq \lambda / \delta, \quad y=x-1, x+1, x \in \mathbb{Z} .
$$

(a) If $\lambda / \delta<2$, then (1.5) holds with $C$ and $\gamma$ as given there.

(b) If, further, $\gamma>2 \ln 2$, then (1.7) holds with $c_{1}$ as given there.

If $\boldsymbol{\lambda}$ and $\boldsymbol{\delta}$ are random sequences satisfying (1.9) with probability one, then parts (a) and (b) are valid a.s.

The situation is more complicated when $\lambda, \delta$ are random but do not a.s. satisfy (1.9) with $\lambda / \delta<2$.

Remark 1.6 The authors acknowledge Massimo Campanino's announcement in a lecture on 12 June 2019 of his perturbative proof with Michele Gianfelice of a version of Theorem 1.2 for sufficiently small $\theta$, using cluster expansions. That announcement stimulated the authors of the current work.

\section{Estimates via the Continuum Random-Cluster Model}

We write $\mathbb{R}$ for the reals and $\mathbb{Z}$ for the integers. The continuum percolation model on $\mathbb{Z} \times \mathbb{R}$ is constructed as in $[20,21]$. For $x \in \mathbb{Z}$, let $D_{x}$ be a Poisson process of points in $\{x\} \times \mathbb{R}$ with 
intensity $\delta$; the processes $\left\{D_{x}: x \in \mathbb{Z}\right\}$ are independent, and the points in the $D_{x}$ are termed 'deaths'. The lines $\{x\} \times \mathbb{R}$ are called 'time lines'.

For $x \in \mathbb{Z}$, let $B_{x}$ be a Poisson process of points in $\left\{x+\frac{1}{2}\right\} \times \mathbb{R}$ with intensity $\lambda$; the processes $\left\{B_{x}: x \in \mathbb{Z}\right\}$ are independent of each other and of the $D_{y}$. For $x \in \mathbb{Z}$ and each $\left(x+\frac{1}{2}, t\right) \in B_{x}$, we draw a unit line-segment in $\mathbb{R}^{2}$ with endpoints $(x, t)$ and $(x+1, t)$, and we refer to this as a 'bridge' joining its two endpoints. For $(x, s),(y, t) \in \mathbb{Z} \times \mathbb{R}$, we write $(x, s) \leftrightarrow(y, t)$ if there exists a path $\pi$ in $\mathbb{R}^{2}$ with endpoints $(x, s),(y, t)$ such that: $\pi$ comprises sub-intervals of $\mathbb{Z} \times \mathbb{R}$ containing no deaths, together possibly with bridges. For $\Lambda, \Delta \subseteq \mathbb{Z} \times \mathbb{R}$, we write $\Lambda \leftrightarrow \Delta$ if there exist $a \in \Lambda$ and $b \in \Delta$ such that $a \leftrightarrow b$. Let $\mathbb{P}_{\Lambda, \lambda, \delta}$ denote the associated probability measure when restricted to the set $\Lambda$, and write $\theta=\lambda / \delta$.

Let $\mathbb{P}_{\lambda, \delta}$ be the corresponding measure on the whole space $\mathbb{Z} \times \mathbb{R}$, and recall from [9, Thm 1.12] that the value $\theta=1$ is the critical point of the continuum percolation model.

The continuum random-cluster model on $\mathbb{Z} \times \mathbb{R}$ is defined as follows. Let $a, b \in \mathbb{Z}, s, t \in \mathbb{R}$ satisfy $a \leq b$ and $s \leq t$, and write $\Lambda=[a, b] \times[s, t]$ for the box $\{a, a+1, \ldots, b\} \times[s, t]$. Its boundary $\partial \Lambda$ is the set of all points $(x, y) \in \Lambda$ such that: either $x \in\{a, b\}$, or $y \in\{s, t\}$, or both.

As sample space we take the set $\Omega_{\Lambda}$ comprising all finite subsets (of $\Lambda$ ) of deaths and bridges, and we assume that no death is the endpoint of any bridge. For $\omega \in \Omega_{\Lambda}$, we write $B(\omega)$ and $D(\omega)$ for the sets of bridges and deaths, respectively, of $\omega$.

The top/bottom periodic boundary condition is imposed on $\Lambda$ : for $x \in[a, b]$, we identify the two points $(x, s)$ and $(x, t)$. The remaining boundary of $\Lambda$, denoted $\partial^{\mathrm{h}} \Lambda$, is the set of points of the form $(x, u) \in \Lambda$ with $x \in\{a, b\}$ and $u \in[s, t]$.

For $\omega \in \Omega_{\Lambda}$, let $k(\omega)$ be the number of its clusters, counted according to the connectivity relation $\leftrightarrow$ (and subject to the above boundary condition). Let $q \in(0, \infty)$, and define the 'continuum random-cluster' probability measure $\mathbb{P}_{\Lambda, \lambda, \delta, q}$ by

$$
d \mathbb{P}_{\Lambda, \lambda, \delta, q}(\omega)=\frac{1}{Z} q^{k(\omega)} d \mathbb{P}_{\Lambda, \lambda, \delta}(\omega), \quad \omega \in \Omega_{\Lambda},
$$

where $Z$ is the appropriate partition function. As at [21, eqn (5.3)],

$$
\mathbb{P}_{\Lambda, \lambda, \delta, q} \leq_{\mathrm{st}} \mathbb{P}_{\Lambda, \lambda, \delta}, \quad q \geq 1,
$$

in the sense of stochastic ordering.

We introduce next a variant in which the box $\Lambda$ possesses a 'slit' at its centre. Let $L \in$ $\{0,1,2, \ldots\}$ and $S_{L}=[0, L] \times\{0\}$. We think of $S_{L}$ as a collection of $L+1$ vertices labelled in the obvious way as $x=0,1,2, \ldots, L$. For $m \geq 2, \beta>0$, let $\Lambda_{m, \beta}$ be the box

$$
\Lambda_{m, \beta}=[-m, m+L] \times\left[-\frac{1}{2} \beta, \frac{1}{2} \beta\right]
$$

subject to a 'slit' along $S_{L}$. That is, $\Lambda_{m, \beta}$ is the usual box except that each vertex $x \in S_{L}$ is replaced by two distinct vertices $x^{+}$and $x^{-}$. The vertex $x^{+}$(respectively, $x^{-}$) is attached to the half-line $\{x\} \times(0, \infty)$ (respectively, the half-line $\{x\} \times(-\infty, 0)$ ); there is no direct connection between $x^{+}$and $x^{-}$. Write $S_{L}^{ \pm}=\left\{x^{ \pm}: x \in S_{L}\right\}$ for the upper and lower sections of the slit $S_{L}$. Henceforth we take $q=2$. Let $\bar{\phi}_{m, \beta}$ be the continuum random-cluster measure on the slit box $\Lambda_{m, \beta}$ with parameters $\lambda, \delta, q=2$ and free boundary condition on $\partial \Lambda_{m, \beta}$, and let $\phi_{m, \beta}$ be the corresponding probability measure with top/bottom periodic boundary condition.

We make a note concerning exponential decay which will be important later. The critical point of the infinite-volume $(q=2)$ continuum random-cluster model on $\mathbb{Z} \times \mathbb{R}$ with parameters $\lambda, \delta$ is given by $\theta_{\mathrm{c}}=2$ where $\theta=\lambda / \delta$ (see [13, Thm 7.1]). Furthermore, as in 
[19, Thm 5.33(b)], there is a unique infinite-volume weak limit, denoted $\phi_{\lambda, \delta}$, when $\theta<2$. In particular (as in the discussion of [13]) there is exponential decay of connectivity when $\theta<2$. Let $\Lambda_{m}=[-m, m]^{2} \subseteq \mathbb{Z} \times \mathbb{R}$, with boundary $\partial \Lambda_{m}$.

Theorem 2.1 ( $\left[13\right.$, Thms 6.2, 7.1]) Let $\lambda, \delta \in(0, \infty)$, and $I=\{0\} \times\left[-\frac{1}{2}, \frac{1}{2}\right] \subseteq \mathbb{Z} \times \mathbb{R}$. There exist $C=C(\lambda, \delta) \in(0, \infty)$ and $\gamma=\gamma(\lambda, \delta)$ satisfying $\gamma>0$ when $\theta=\lambda / \delta<2$, such that

$$
\phi_{\lambda, \delta}\left(I \leftrightarrow \partial \Lambda_{m}\right) \leq C e^{-\gamma m}, \quad m \geq 0 .
$$

The function $\gamma(\lambda, \delta)$ may be chosen to satisfy $\gamma \rightarrow \infty$ as $\delta \rightarrow \infty$ for fixed $\lambda$.

Henceforth the function $\gamma$ denotes that of Theorem 2.1. (The function $\gamma$ in Theorems 1.2, 1.4 is derived from that of Theorem 2.1.) By stochastic domination, (2.3) holds with $\phi_{\lambda, \delta}$ replaced by $\mathbb{P}_{\Lambda, \lambda, \delta, 2}$ for general boxes $\Lambda$.

It is explained in [21] that a random-cluster configuration $\omega$ gives rise, by a clusterlabelling process, to an Ising configuration on $\Lambda$, which serves (see [2]) as a two-dimensional representation of the quantum Ising model of (1.2). We shall use $\bar{\phi}_{m, \beta}$ and $\phi_{m, \beta}$ to denote the respective couplings of the continuum random-cluster measures and the corresponding (Ising) spin-configurations, and $\bar{\phi}_{m, \beta}^{\eta}, \phi_{m, \beta}^{\eta}$ for the measures with spin-configuration $\eta$ on $\partial^{\mathrm{h}} \Lambda_{m, \beta}$.

Remark 2.2 Theorem 2.1 is an important component of the estimates that follow. At the time of the writing of [21], the result was known only when $\theta<1$, and the corresponding exponential-decay theorem [21, Thm 6.7] was proved by stochastic comparison with continuum percolation (see (2.2)). More recent progress of [13] has allowed its extension to the $q=2$ continuum random-cluster model directly. In order to apply it in the current work, a minor extension of the ratio weak-mixing theorem [21, Thm 7.1] is needed, namely that the mixing theorem holds with $\bar{\phi}$ taken to be the random-cluster measure on $\Lambda$ with free boundary conditions. The proof is unchanged.

Remark 2.3 In the proofs that follow, it would be convenient to have a stronger version of (2.3) with $\phi_{\lambda, \delta}$ replaced by the finite-volume random-cluster measure on $\Lambda_{m, \beta}$ with wired boundary condition on $\partial^{\mathrm{h}} \Lambda_{m, \beta}$ and periodic top/bottom boundary condition. It may be possible to derive such an inequality as in [16], but we do not pursue that option here.

Remark 2.4 We shall work only in the subcritical phase $\theta=\lambda / \delta<2$. As remarked prior to Theorem 2.1, there exists a unique infinite-volume measure. Similarly, the limits

$$
\bar{\phi}_{m}=\lim _{\beta \rightarrow \infty} \bar{\phi}_{m, \beta}, \quad \phi_{m}=\lim _{\beta \rightarrow \infty} \phi_{m, \beta},
$$

exist and are identical measures on the strip $\Lambda_{m}=[-m, m] \times(-\infty, \infty)$.

Let $\Omega_{m, \beta}$ be the sample space of the continuum random-cluster model on $\Lambda_{m, \beta}$, and $\Sigma_{m, \beta}$ the set of admissible allocations of spins to the clusters of configurations, as in [21, Sect. 5]. For $\sigma \in \Sigma_{m, \beta}$ and $x \in S_{L}$, write $\sigma_{x}^{ \pm}$for the spin-state of $x^{ \pm}$. Let $\Sigma_{L}=\{-1,+1\}^{L+1}$ be the set of spin-configurations of the vectors $\left\{x^{+}: x \in S_{L}\right\}$ and $\left\{x^{-}: x \in S_{L}\right\}$, and write $\sigma_{L}^{+}=\left(\sigma_{x}^{+}: x \in S_{L}\right)$ and $\sigma_{L}^{-}=\left(\sigma_{x}^{-}: x \in S_{L}\right)$.

Let

$$
a_{m, \beta}=\bar{\phi}_{m, \beta}\left(\sigma_{L}^{+}=\sigma_{L}^{-}\right) \text {. }
$$

Then,

$$
a_{m, \beta} \rightarrow a_{m}=\phi_{m}\left(\sigma_{L}^{+}=\sigma_{L}^{-}\right) \quad \text { as } \beta \rightarrow \infty,
$$


where $\phi_{m}=\lim _{\beta \rightarrow \infty} \phi_{m, \beta}$ as in Remark 2.4.

Here is the main estimate of this section, of which Theorem 1.2 is an immediate corollary with adapted values of the constants. It differs from [22, Thm 6.5] in the removal of a factor of order $L^{\alpha}$, and the replacement of the condition $\theta<1$ by the weaker assumption $\theta<2$.

Theorem 2.5 Let $\lambda, \delta \in(0, \infty)$ and write $\theta=\lambda / \delta$. If $\theta<2$, there exist $C, M \in(0, \infty)$, depending on $\theta$ only, such that the following holds. For $L \geq 1$ and $M \leq m \leq n<\infty$,

$$
\sup _{\|c\|=1}\left|\frac{\phi_{m}\left(c\left(\sigma_{L}^{+}\right) c\left(\sigma_{L}^{-}\right)\right)}{a_{m}}-\frac{\phi_{n}\left(c\left(\sigma_{L}^{+}\right) c\left(\sigma_{L}^{-}\right)\right)}{a_{n}}\right| \leq C e^{-\frac{1}{3} \gamma m},
$$

where $\gamma$ is as in Theorem 2.1, and the supremum is over all functions $c: \Sigma_{L} \rightarrow \mathbb{R}$ with $L^{2}$-norm satisfying $\|c\|=1$.

In the proof of Theorem 2.5, we make use of the following two lemmas (corresponding, respectively, to [21, Lemmas 6.8, 6.9]), which are proved in Sect. 3 using the method of ratio weak-mixing.

Lemma 2.6 Let $\lambda, \delta \in(0, \infty)$ satisfy $\theta=\lambda / \delta<2$, and let $\gamma$ be as in Theorem 2.1. There exist constants $A(\lambda, \delta), C_{1}(\lambda, \delta) \in(0, \infty)$ such that the following holds. Let

$$
R_{K}=C_{1} e^{-\frac{1}{2} \gamma K} .
$$

For all $L \geq 3,1 \leq K<\frac{1}{2} L, m \geq 1, \beta \geq 1$, and all $\epsilon^{+}, \epsilon^{-} \in \Sigma_{L}$, we have that

$$
A^{2 K}\left(1-R_{K}\right) \leq \frac{\bar{\phi}_{m, \beta}\left(\sigma_{L}^{+}=\epsilon^{+}, \sigma_{L}^{-}=\epsilon^{-}\right)}{\bar{\phi}_{m, \beta}\left(\sigma_{L}^{+}=\epsilon^{+}\right) \bar{\phi}_{m, \beta}\left(\sigma_{L}^{-}=\epsilon^{-}\right)} \leq A^{-2 K}\left(1+R_{K}\right),
$$

whenever $K$ is such that $R_{K} \leq \frac{1}{2}$.

In the second lemma we allow a general spin boundary condition on $\partial^{\mathrm{h}} \Lambda_{m, \beta}$.

Lemma 2.7 Let $\lambda, \delta \in(0, \infty)$ satisfy $\theta=\lambda / \delta<2$, and let $\gamma$ be as in Theorem 2.1 . There exists a constant $C_{1} \in(0, \infty)$ such that: for all $L \geq 3, m \geq 1, \beta \geq 1$, all events $A \subseteq \Sigma_{L} \times \Sigma_{L}$, and all admissible spin boundary conditions $\eta$ of $\partial^{\mathrm{h}} \Lambda_{m, \beta}$,

$$
\left|\frac{\bar{\phi}_{m, \beta}^{\eta}\left(\left(\sigma_{L}^{+}, \sigma_{L}^{-}\right) \in A\right)}{\bar{\phi}_{m, \beta}\left(\left(\sigma_{L}^{+}, \sigma_{L}^{-}\right) \in A\right)}-1\right| \leq C_{1} e^{-\frac{2}{7} \gamma m},
$$

whenever the right side of the inequality is less than 1.

Proof of Theorem 2.5 Let $\theta<2$, and let $\gamma$ be as in Theorem 2.1. It suffices to prove (2.7) with $\phi_{m}$ (respectively, $\phi_{n}$ ) replaced by $\bar{\phi}_{m, \beta}$ (respectively, $\bar{\phi}_{n, \beta}$ ), and $a_{m}$ (respectively, $a_{n}$ ) replaced by $a_{m, \beta}$ (respectively, $a_{n, \beta}$ ). Having done so, we let $\beta \rightarrow \infty$ to obtain (2.7) by Remark 2.4.

Let $A, C_{1}, R_{K}$ be as in Lemma 2.6, and let $L \geq 3$ and $1 \leq K<\frac{1}{2} L$ be such that

$$
R_{K} \leq \frac{1}{4}
$$

Remaining small values of $L$ are covered in (2.7) by adjusting $C$.

Since $\bar{\phi}_{m, \beta} \leq_{\text {st }} \bar{\phi}_{n, \beta}$, we may couple $\bar{\phi}_{m, \beta}$ and $\bar{\phi}_{n, \beta}$ via a probability measure $v$ on pairs $\left(\omega_{1}, \omega_{2}\right)$ of configurations on $\Lambda_{n, \beta}$ in such a way that $v\left(\omega_{1} \leq \omega_{2}\right)=1$. It is standard (as in $[19,24])$ that we may find $v$ such that $\omega_{1}$ and $\omega_{2}$ are identical configurations within the 


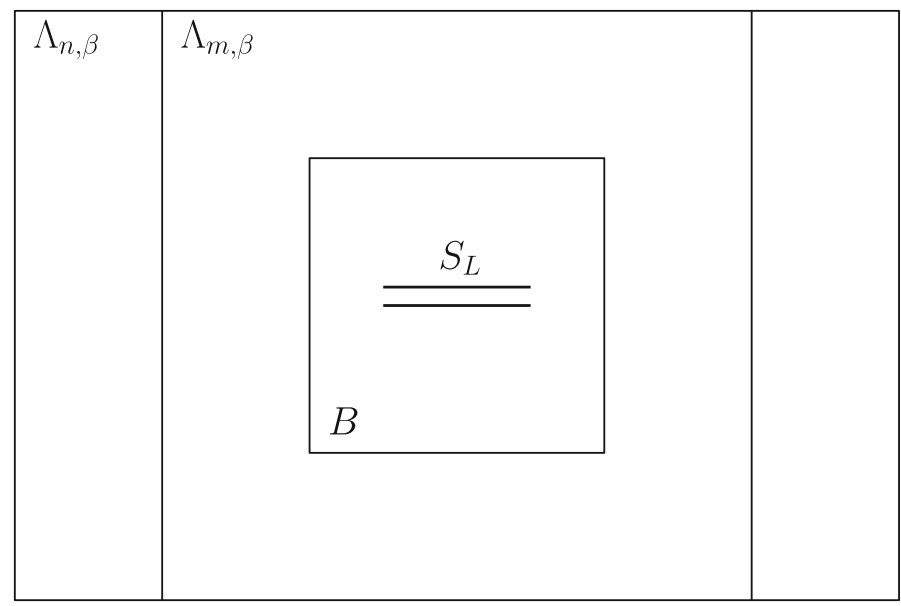

Fig. 1 The boxes $\Lambda_{n, \beta}, \Lambda_{m, \beta}$, and $B$

region of $\Lambda_{m, \beta}$ that is not connected to $\partial^{\mathrm{h}} \Lambda_{m, \beta}$ in the upper configuration $\omega_{2}$. Let $D$ be the set of all pairs $\left(\omega_{1}, \omega_{2}\right) \in \Omega_{n, \beta} \times \Omega_{n, \beta}$ such that: $\omega_{2}$ contains no path joining $\partial B$ to $\partial^{\mathrm{h}} \Lambda_{m, \beta}$, where

$$
B=[-r, r+L] \times[-r, r], \quad r=\left\lfloor\frac{1}{2} m\right\rfloor .
$$

The relevant regions are illustrated in Fig. 1.

Having constructed the measure $v$ accordingly, we may now allocate spins to the clusters of $\omega_{1}$ and $\omega_{2}$ in the manner described in [21, Sect. 5]. This may be done in such a way that, on the event $D$, the spin-configurations associated with $\omega_{1}$ and $\omega_{2}$ within $B$ are identical. We write $\sigma_{1}$ (respectively, $\sigma_{2}$ ) for the spin-configuration on the clusters of $\omega_{1}$ (respectively, $\omega_{2}$ ), and $\sigma_{i, L}^{ \pm}$for the spins of $\sigma_{i}$ on the slit $S_{L}$.

By the remark following [21, Eq. (6.4)], it suffices to consider non-negative functions $c: \Sigma_{L} \rightarrow \mathbb{R}$, and thus we let $c: \Sigma_{L} \rightarrow[0, \infty)$ with $\|c\|=1$. Let

$$
S_{c}=\frac{c\left(\sigma_{1, L}^{+}\right) c\left(\sigma_{1, L}^{-}\right)}{a_{m, \beta}}-\frac{c\left(\sigma_{2, L}^{+}\right) c\left(\sigma_{2, L}^{-}\right)}{a_{n, \beta}},
$$

so that

$$
\frac{\bar{\phi}_{m, \beta}\left(c\left(\sigma_{L}^{+}\right) c\left(\sigma_{L}^{-}\right)\right)}{a_{m, \beta}}-\frac{\bar{\phi}_{n, \beta}\left(c\left(\sigma_{L}^{+}\right) c\left(\sigma_{L}^{-}\right)\right)}{a_{n, \beta}}=v\left(S_{c} 1_{D}\right)+v\left(S_{c} 1 \bar{D}\right),
$$

where $\bar{D}$ is the complement of $D$, and $1_{E}$ is the indicator function of $E$.

Consider first the term $v\left(S_{c} 1_{D}\right)$ in (2.12). On the event $D$, we have that $\sigma_{1, L}^{ \pm}=\sigma_{2, L}^{ \pm}$, so that

$$
\left|v\left(S_{c} 1_{D}\right)\right| \leq\left|1-\frac{a_{m, \beta}}{a_{n, \beta}}\right| \frac{\bar{\phi}_{m, \beta}\left(c\left(\sigma_{L}^{+}\right) c\left(\sigma_{L}^{-}\right)\right)}{a_{m, \beta}} .
$$

By Lemma 2.6 and [21, Lemma 6.10],

$$
\begin{aligned}
\bar{\phi}_{m, \beta}\left(c\left(\sigma_{L}^{+}\right) c\left(\sigma_{L}^{-}\right)\right) & =\sum_{\epsilon^{ \pm} \in \Sigma_{L}} c\left(\epsilon^{+}\right) c\left(\epsilon^{-}\right) \bar{\phi}_{m, \beta}\left(\sigma_{L}^{+}=\epsilon^{+}, \sigma_{L}^{-}=\epsilon^{-}\right) \\
& \leq A^{-2 K}\left(1+R_{K}\right) \bar{\phi}_{m, \beta}\left(c\left(\sigma_{L}^{+}\right)\right) \bar{\phi}_{m, \beta}\left(c\left(\sigma_{L}^{-}\right)\right)
\end{aligned}
$$




$$
\begin{aligned}
& =A^{-2 K}\left(1+R_{K}\right)\left(\sum_{\epsilon \in \Sigma_{L}} c(\epsilon) \bar{\phi}_{m, \beta}\left(\sigma_{L}^{+}=\epsilon\right)\right)^{2} \\
& \leq A^{-2 K}\left(1+R_{K}\right) \sum_{\epsilon \in \Sigma_{L}} \bar{\phi}_{m, \beta}\left(\sigma_{L}^{+}=\epsilon\right)^{2},
\end{aligned}
$$

where we have used reflection-symmetry in the horizontal axis at the intermediate step. By Lemma 2.6 and reflection-symmetry again,

$$
\begin{aligned}
a_{m, \beta} & =\sum_{\epsilon \in \Sigma_{L}} \bar{\phi}_{m, \beta}\left(\sigma_{L}^{+}=\sigma_{L}^{-}=\epsilon\right) \\
& \geq A^{2 K}\left(1-R_{K}\right) \sum_{\epsilon \in \Sigma_{L}} \bar{\phi}_{m, \beta}\left(\sigma_{L}^{+}=\epsilon\right)^{2} .
\end{aligned}
$$

Therefore,

$$
\frac{\bar{\phi}_{m, \beta}\left(c\left(\sigma_{L}^{+}\right) c\left(\sigma_{L}^{-}\right)\right)}{a_{m, \beta}} \leq A^{-4 K} \frac{1+R_{K}}{1-R_{K}} .
$$

We set $A=\left\{\sigma_{L}^{+}=\sigma_{L}^{-}\right\}$in Lemma 2.7 to find that, for sufficiently large $m \geq M_{1}(\lambda, \delta)$,

$$
\left|\frac{\bar{\phi}_{m, \beta}^{\eta}\left(\sigma_{L}^{+}=\sigma_{L}^{-}\right)}{\bar{\phi}_{m, \beta}\left(\sigma_{L}^{+}=\sigma_{L}^{-}\right)}-1\right| \leq C e^{-\frac{2}{7} \gamma m}<\frac{1}{2} .
$$

Each of the two probabilities on the left side may be interpreted as probabilities in the continuum Potts model of [21, Eq. (5.4)] on $\Lambda_{m}$. By averaging over $\eta$, sampled according to $\bar{\phi}_{n, \beta}$ when viewed as a Potts measure, we deduce by the spatial Markov property that

$$
\left|\frac{\bar{\phi}_{n, \beta}\left(\sigma_{L}^{+}=\sigma_{L}^{-}\right)}{\bar{\phi}_{m, \beta}\left(\sigma_{L}^{+}=\sigma_{L}^{-}\right)}-1\right| \leq C e^{-\frac{2}{7} \gamma m}<\frac{1}{2},
$$

which is to say that

$$
\left|\frac{a_{n, \beta}}{a_{m, \beta}}-1\right| \leq C e^{-\frac{2}{7} \gamma m}<\frac{1}{2} .
$$

We make a note for later use. In the same way as above, a version of inequality (2.15) holds with $\bar{\phi}_{m, \beta}$ replaced by the continuum random-cluster measure $\bar{\phi}_{B}$ on the box $B$ with free boundary conditions, namely,

$$
\frac{\bar{\phi}_{B}\left(c\left(\sigma_{L}^{+}\right) c\left(\sigma_{L}^{-}\right)\right)}{a_{B}} \leq A^{-4 K} \frac{1+R_{K}}{1-R_{K}},
$$

where $a_{B}=\bar{\phi}_{B}\left(\sigma_{L}^{+}=\sigma_{L}^{-}\right)$. By (2.10) and (2.16), we may take $C$ and $M_{1}$ above such that

$$
\left|\frac{a_{n, \beta}}{a_{B}}-1\right| \leq C e^{-\frac{1}{7} \gamma m}<\frac{1}{2}, \quad m \geq M_{1}(\lambda, \delta) .
$$

Inequalities (2.15) and (2.16) may be combined as in (2.13) to obtain

$$
\left|v\left(S_{C} 1_{D}\right)\right| \leq C_{1} A^{-4 K} \frac{1+R_{K}}{1-R_{K}} e^{-\frac{2}{7} \gamma m}
$$

for an appropriate constant $C_{1}=C_{1}(\lambda, \delta)$ and all $m \geq M_{1}$. 
We turn to the term $v\left(S_{c} 1 \bar{D}\right)$ in (2.12). Evidently,

$$
\left|v\left(S_{c} 1 \bar{D}\right)\right| \leq A_{m}+B_{n},
$$

where

$$
A_{m}=\frac{v\left(c\left(\sigma_{1, L}^{+}\right) c\left(\sigma_{1, L}^{-}\right) 1 \bar{D}\right)}{a_{m, \beta}}, \quad B_{n}=\frac{v\left(c\left(\sigma_{2, L}^{+}\right) c\left(\sigma_{2, L}^{-}\right) 1 \bar{D}\right)}{a_{n, \beta}} .
$$

There exist constants $C_{2}, M_{2}$ depending on $\lambda, \delta$, such that, for $m>r \geq M_{2}$,

$$
\begin{aligned}
B_{n} & =\frac{v(\bar{D})}{a_{n, \beta}} v\left(c\left(\sigma_{2, L}^{+}\right) c\left(\sigma_{2, L}^{-}\right) \mid \bar{D}\right) \\
& =\frac{v(\bar{D})}{a_{n, \beta}} \bar{\phi}_{n, \beta}\left(\bar{\phi}_{B}^{\eta}\left(c\left(\sigma_{2, L}^{+}\right) c\left(\sigma_{2, L}^{-}\right)\right) \mid \bar{D}\right) \\
& \leq \frac{v(\bar{D})}{a_{B}} C_{2} \bar{\phi}_{B}\left(c\left(\sigma_{L}^{+}\right) c\left(\sigma_{L}^{-}\right)\right)
\end{aligned}
$$

by Lemma 2.7 with $\bar{\phi}_{m, \beta}$ replaced by $\bar{\phi}_{B}$, and (2.18). At the middle step, we have used conditional expectation given the spin configuration $\eta$ on $\Lambda_{m, \beta} \backslash B$. By (2.17),

$$
B_{n} \leq v(\bar{D}) A^{-4 K} \frac{1+R_{K}}{1-R_{K}} .
$$

A similar upper bound is valid for $A_{m}$, on noting that the conditioning on $\bar{D}$ imparts certain information about the configuration $\omega_{1}$ outside $B$ but nothing further about $\omega_{1}$ within $B$. Combining this with (2.20)-(2.22), we find that, for $r \geq M_{3}(\lambda, \delta)$ and some $C_{3}=C_{3}(\lambda, \delta)$,

$$
\left|v\left(S_{c} 1 \bar{D}\right)\right| \leq v(\bar{D}) C_{3} A^{-4 K} \frac{1+R_{K}}{1-R_{K}} .
$$

By (2.2), (2.10), and Theorem 2.1,

$$
v(\bar{D}) \leq C_{4} m e^{-\frac{1}{2} \gamma m} \leq C_{5} e^{-\frac{1}{3} \gamma m}, \quad m \geq M_{4},
$$

for some $C_{4}, C_{5}, M_{4} \geq 2 M_{3}$. We combine (2.19), (2.23), (2.24) as in (2.12). Letting $\beta \rightarrow \infty$ and recalling (2.9), we obtain (2.7) from (2.6), for $m \geq M:=\max \left\{M_{1}, M_{2}, M_{4}\right\}$.

Finally, we remark that $C$ and $M$ depend on both $\lambda$ and $\delta$. The left side of (2.7) is invariant under re-scalings of the time-axes, that is, under the transformations $(\lambda, \delta) \mapsto(\lambda \eta, \delta \eta)$ for $\eta \in(0, \infty)$. We may therefore work with the new values $\lambda^{\prime}=\theta, \delta^{\prime}=1$, with appropriate constants $\alpha(\theta, 1), C(\theta, 1), M(\theta, 1)$.

\section{Proofs of Lemmas 2.6 and 2.7}

Let $\Lambda$ be a box in $\mathbb{Z} \times \mathbb{R}$ (we shall later consider a box $\Lambda$ with a slit $S_{L}$, for which the same definitions and results are valid). A path $\pi$ of $\Lambda$ is an alternating sequence of disjoint intervals (contained in $\Lambda$ ) and unit line-segments of the form $\left[z_{0}, z_{1}\right], b_{12},\left[z_{2}, z_{3}\right], b_{34}, \ldots$, $b_{2 k-1,2 k},\left[z_{2 k}, z_{2 k+1}\right]$, where: each pair $z_{2 i}, z_{2 i+1}$ is on the same time line of $\Lambda$, and $b_{2 i-1,2 i}$ is a unit line-segment with endpoints $z_{2 i-1}$ and $z_{2 i}$, perpendicular to the time-lines. The path $\pi$ is said to join $z_{0}$ and $z_{2 k+1}$. The length of $\pi$ is its one-dimensional Lebesgue measure. A circuit $D$ of $\Lambda$ is a path except inasmuch as $z_{0}=z_{2 k+1}$. A set $D$ is called linear if it is a disjoint union of paths and/or circuits. Let $\Delta, \Gamma$ be disjoint subsets of $\Lambda$. The linear set $D$ is 
said to separate $\Delta$ and $\Gamma$ if every path of $\Lambda$ from $\Delta$ to $\Gamma$ passes through $D$, and $D$ is minimal with this property in that no strict subset of $D$ has the property.

Let $\omega \in \Omega_{\Lambda}$. An open path $\pi$ of $\omega$ is a path of $\Lambda$ such that, in the notation above, the intervals $\left[z_{2 i}, z_{2 i+1}\right]$ contain no death of $\omega$, and the line-segments $b_{2 i-1,2 i}$ are bridges of $\omega$.

Let $\Gamma$ be a measurable subset and $\Delta$ a finite subset of $\Lambda$ such that $\Delta \cap \Gamma=\varnothing$. We shall make use of the 'ratio weak-mixing property' of the spin-configurations in $\Delta$ and $\Gamma$ that is stated and proved in [21, Thm 7.1]; note Remark 2.2.

Consider the box $\Lambda_{m, \beta}$ with slit $S_{L}$. Let $K$ be an integer satisfying $1 \leq K<\frac{1}{2} L$, and let

$$
\begin{aligned}
& \Delta=\left\{x^{+}: x \in S_{L}, K \leq x \leq L-K\right\}, \\
& \Gamma=\left\{x^{-}: x \in S_{L}, K \leq x \leq L-K\right\} .
\end{aligned}
$$

The following replaces [21, Lemma 7.24].

Lemma 3.1 Let $\lambda, \delta \in(0, \infty)$ satisfy $\theta=\lambda / \delta<2$, and let $\gamma>0$ be as in Theorem 2.1. There exists $C_{1}=C_{1}(\lambda, \delta) \in(0, \infty)$ such that the following holds. For $\epsilon_{K}^{+} \in \Sigma_{\Delta}, \epsilon_{K}^{-} \in \Sigma_{\Gamma}$, we have that

$$
\left|\frac{\bar{\phi}_{m, \beta}\left(\sigma_{\Delta}=\epsilon_{K}^{+}, \sigma_{\Gamma}=\epsilon_{K}^{-}\right)}{\bar{\phi}_{m, \beta}\left(\sigma_{\Delta}=\epsilon_{K}^{+}\right) \bar{\phi}_{m, \beta}\left(\sigma_{\Gamma}=\epsilon_{K}^{-}\right)}-1\right| \leq C_{1} e^{-\frac{1}{2} \gamma K},
$$

whenever the right side is less than $\frac{1}{2}$.

Proof Take

$$
D=([-m, 0) \times\{0\}) \cup((L, L+m] \times\{0\}),
$$

the union of the two horizontal line-segments that, when taken with the slit $S_{L}$, complete the 'equator' of $\Lambda_{m, \beta}$. Thus $D$ is a linear subset of $\Lambda_{m, \beta}$ that separates $\Delta$ and $\Gamma$. Let $t_{1}, t_{2}, t$ be as in [21, Thm 7.1], namely,

$$
\begin{gathered}
t_{1}=\bar{\phi}_{m, \beta}(\Delta \leftrightarrow D), \quad t_{2}=\sqrt{\bar{\phi}_{m, \beta}(D \leftrightarrow \Gamma),} \\
t=t_{1}+2 t_{2}+\frac{t_{1}+t_{2}}{1-t_{1}-2 t_{2}} .
\end{gathered}
$$

By Theorem 2.1, there exist constants $C_{2}, C_{3}$, depending on $\lambda$ and $\delta$ only, such that

$$
t_{1} \leq 2 \sum_{i=K}^{\lfloor L / 2\rfloor} C_{2} e^{-\gamma i} \leq C_{3} e^{-\gamma K},
$$

and furthermore $t_{2}^{2}=t_{1}$. The claim now follows by [21, Thm 7.1] and Remark 2.2.

We now prove Lemmas 2.6 and 2.7 .

Proof of Lemma 2.6 Let $\theta<2$ and let $\gamma$ be as in Theorem 2.1. With $1 \leq K<\frac{1}{2} L$, write $\sigma_{L, K}^{ \pm}=\left(\sigma_{x}^{ \pm}: K \leq x \leq L-K\right)$. First, let $x=(L, 0)$, and let $\epsilon^{+}, \epsilon^{-} \in\{-1,+1\}^{L+1}$ be possible spin-vectors of the sets $S_{L}^{+}$and $S_{L}^{-}$, respectively. By [21, Lemma 7.25] with $S=S_{L}^{+} \cup S_{L}^{-} \backslash\left\{x^{+}\right\}$,

$$
\begin{aligned}
& \bar{\phi}_{m, \beta}\left(\sigma_{L}^{+}=\epsilon^{+}, \sigma_{L}^{-}=\epsilon^{-}\right) \\
& \quad \geq \frac{1}{2} \bar{\phi}_{m, \beta}\left(\sigma_{y}^{+}=\epsilon_{y}^{+} \text {for } y \in S_{L}^{+} \backslash\left\{x^{+}\right\}, \sigma_{L}^{-}=\epsilon^{-}\right) \mathbb{P}_{\Lambda_{m, \beta}, \lambda, \delta}\left(x^{+} \leftrightarrow S\right) .
\end{aligned}
$$


Now, $\mathbb{P}_{\Lambda_{m, \beta}, \lambda, \delta}(x \leftrightarrow S)$ is at least as large as the probability that the first event (death or bridge) encountered on moving northwards from $x$ is a death, so that

$$
\mathbb{P}_{\Lambda_{m, \beta}, \lambda, \delta}(x \leftrightarrow S) \geq \frac{\delta}{2 \lambda+\delta} .
$$

On iterating the above, we obtain that

$$
\bar{\phi}_{m, \beta}\left(\sigma_{L}^{+}=\epsilon^{+}, \sigma_{L}^{-}=\epsilon^{-}\right) \geq A^{2 K} \bar{\phi}_{m, \beta}\left(\sigma_{L, K}^{+}=\epsilon_{K}^{+}, \sigma_{L, K}^{-}=\epsilon_{K}^{-}\right),
$$

where $\epsilon_{K}^{ \pm}$is the vector obtained from $\epsilon^{ \pm}$by removing the entries labelled by vertices $x$ satisfying $0 \leq x<K$ and $L-K<x \leq L$, and

$$
A=\left(\frac{\delta}{2(2 \lambda+\delta)}\right)^{2} \text {. }
$$

In summary, for $\epsilon^{ \pm} \in \Sigma_{L}$,

$$
\begin{aligned}
A^{2 K} \bar{\phi}_{m, \beta}\left(\sigma_{L, K}^{+}=\epsilon_{K}^{+}, \sigma_{L, K}^{-}=\epsilon_{K}^{-}\right) & \leq \bar{\phi}_{m, \beta}\left(\sigma_{L}^{+}=\epsilon^{+}, \sigma_{L}^{-}=\epsilon^{-}\right) \\
& \leq \bar{\phi}_{m, \beta}\left(\sigma_{L, K}^{+}=\epsilon_{K}^{+}, \sigma_{L, K}^{-}=\epsilon_{K}^{-}\right) .
\end{aligned}
$$

With $\Delta, \Gamma$ as in (3.1), we apply Lemma 3.1 to obtain that there exists $C_{1}=C_{1}(\lambda, \delta)<\infty$ such that

$$
\left|\frac{\bar{\phi}_{m, \beta}\left(\sigma_{L, K}^{+}=\epsilon_{K}^{+}, \sigma_{L, K}^{-}=\epsilon_{K}^{-}\right)}{\bar{\phi}_{m, \beta}\left(\sigma_{L, K}^{+}=\epsilon_{K}^{+}\right) \bar{\phi}_{m, \beta}\left(\sigma_{L, K}^{-}=\epsilon_{K}^{-}\right)}-1\right| \leq C_{1} e^{-\frac{1}{2} \gamma K},
$$

whenever the right side is less than or equal to $\frac{1}{2}$.

By a similar argument to (3.5),

$$
A^{K} \bar{\phi}_{m, \beta}\left(\sigma_{L, K}^{ \pm}=\epsilon_{K}^{ \pm}\right) \leq \bar{\phi}_{m, \beta}\left(\sigma_{L}^{ \pm}=\epsilon^{ \pm}\right) \leq \bar{\phi}_{m, \beta}\left(\sigma_{L, K}^{ \pm}=\epsilon_{K}^{ \pm}\right) .
$$

The claim follows on combining (3.5)-(3.7).

Proof of Lemma 2.7 Let $\Delta=S_{L}^{+} \cup S_{L}^{-}$and $\Gamma=\partial^{\mathrm{h}} \Lambda_{m, \beta}$, and suppose $\theta<2$. Let $k=\frac{3}{7} m$ and assume for simplicity that $k$ is an integer. (If either $m$ is small or $k$ is non-integral, the constant $C$ may be adjusted accordingly.) Let $D_{0}$ be the circuit illustrated in Fig. 2, comprising a path in the upper half-plane from $(-k, 0)$ to $(L+k, 0)$ together with its reflection in the $x$-axis. Let $D=D_{0} \cap \Lambda_{m, \beta}$. Thus, $D=D_{0}$ in the case $\beta=\beta_{2}$ of the figure. In the case $\beta=\beta_{1}, D$ comprises two disjoint paths of $\Lambda_{m, \beta}$. In each case, $D$ separates $\Delta$ and $\Sigma$.

Let $t_{1}, t_{2}, t$ be as in (3.2). By the ratio weak-mixing theorem [21, Thm 7.1] and Remark 2.2,

$$
\left|\frac{\bar{\phi}_{m, \beta}^{\eta}\left(\left(\sigma_{L}^{+}, \sigma_{L}^{-}\right)=\left(\epsilon^{+}, \epsilon^{-}\right)\right)}{\bar{\phi}_{m, \beta}\left(\left(\sigma_{L}^{+}, \sigma_{L}^{-}\right)=\left(\epsilon^{+}, \epsilon^{-}\right)\right)}-1\right| \leq 2 t, \quad \epsilon^{ \pm} \in \Sigma_{L},
$$

whenever $t \leq \frac{1}{2}$. We multiply up, and sum over $\left(\epsilon^{+}, \epsilon^{-}\right) \in A$ to obtain

$$
\left|\frac{\bar{\phi}_{m, \beta}^{\eta}\left(\sigma_{\Delta} \in A\right)}{\bar{\phi}_{m, \beta}\left(\sigma_{\Delta} \in A\right)}-1\right| \leq 2 t \text {, }
$$

whenever $t \leq \frac{1}{2}$.

By Theorem 2.1, there exist $C_{2}, C_{3}, c_{4}>0$, depending on $\lambda, \delta$, such that

$$
t_{1} \leq 4 \sum_{i=0}^{\lfloor L / 2\rfloor} \bar{\phi}_{m, \beta}\left((i, 0) \leftrightarrow D_{0}\right)
$$




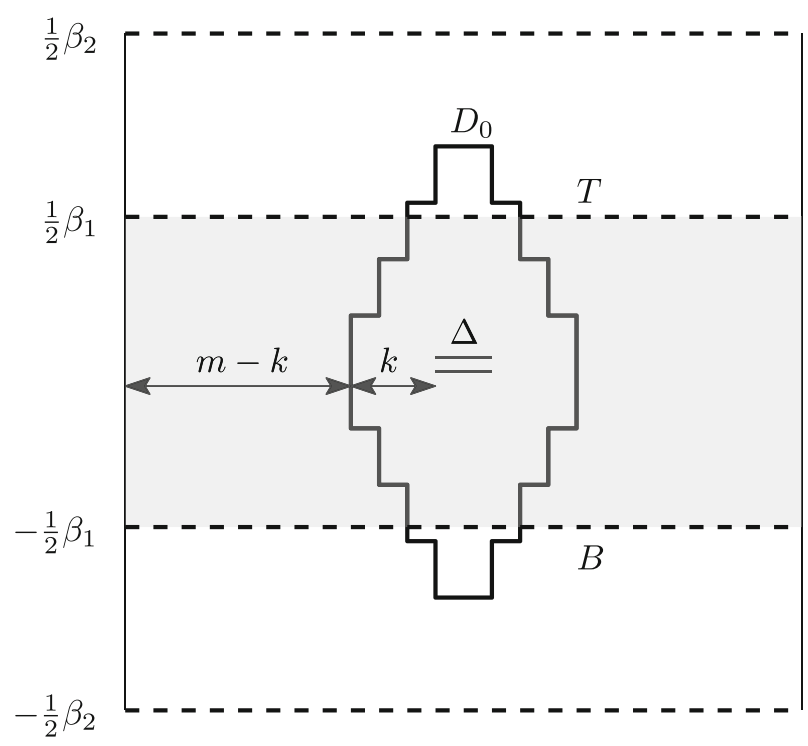

Fig. 2 The circuit $D_{0}$ is approximately a parallelogram with $\Delta$ at its centre. The sides comprise vertical steps of height 2 followed by horizontal steps of length 1 . The horizontal and vertical diagonals of $D_{0}$ have lengths $2 k+L$ and (approximately) $4 k+2 L$ respectively, where $k=\frac{3}{7} m$. Two values of $\beta$ are indicated. When $\beta=\beta_{2}, D_{0}$ is contained in $\Lambda_{m, \beta}$ and we take $D=D_{0}$. When $\beta=\beta_{1}, \Lambda_{m, \beta}$ is the shaded area only, and we work with $D=D_{0} \cap \Lambda_{m, \beta}$ considered as the union of two disjoint paths that separates $\Delta$ and $\Sigma$

$$
\leq 4 \sum_{i=0}^{\lfloor L / 2\rfloor} C_{2} e^{-\gamma \frac{2}{3}(k+i)} \leq C_{3} e^{-\frac{2}{7} \gamma m},
$$

and similarly,

$$
t_{2}^{2} \leq 8 \sum_{i=0}^{\lceil k+L / 2\rceil} C_{2} e^{-\gamma\left(\frac{4}{7} m+c_{4} i\right)} \leq C_{3} e^{-\frac{4}{7} \gamma m} .
$$

The claim follows.

\section{Quenched Disorder}

The parameters $\lambda$ and $\delta$ have so far been assumed constant. The situation is more complicated in the disordered case, when either they vary deterministically, or they are random. The arguments of this paper may be applied in both cases, and the outcomes are summarised in this section. Let the Hamiltonian (1.2) be replaced by (1.8), and write $\lambda=\left(\lambda_{x, x+1}: x \in \mathbb{Z}\right)$ and $\delta=\left(\delta_{x}: x \in \mathbb{Z}\right)$.

The fundamental bound of Theorem 2.5 depends only on the ratio $\theta=\lambda / \delta$. In the disordered setting, the connection probabilities of the continuum random-cluster model are increasing in $\lambda$ and decreasing in $\delta$, and powers of the function $A(\lambda, \delta)$ of $(3.4)$ are replaced by products of the form

$$
A_{x, k}^{\prime}=\prod_{i=1}^{k}\left(\frac{\delta_{x+i}}{2\left(\delta_{x+i}+\lambda_{x+i, x+i-1}+\lambda_{x+i, x+i+1}\right)}\right),
$$


which are decreasing in $\lambda$ and increasing in $\delta$. By examination of the earlier lemmas and proofs, the conclusions of the paper are found to be valid with $\gamma=\gamma(\lambda, \delta)$ whenever (1.9) holds with some $\lambda, \delta>0$. Hence, in the disordered case where (1.9) holds with probability one, the corresponding conclusions are valid a.s. (subject to appropriate bounds on the ratio $\lambda / \delta)$. This proves Theorem 1.5.

Consider now the situation in which (1.9) does not hold with probability one. Suppose that the $\lambda_{x, x+1}, x \in \mathbb{Z}$, are independent, identically distributed random variables, and similarly the $\delta_{x}, x \in \mathbb{Z}$, and assume that the vectors $\lambda$ and $\delta$ are independent. We write $P$ for the corresponding probability measure, viewed as the measure governing the 'random environment'.

A quenched area law might assert something along the following lines: subject to suitable conditions, there exists a random variable $Z$ which is $P$-a.s. finite such that $S\left(\rho_{m}^{L}\right)<Z$ for all appropriate $m, L$. Such a uniform upper bound will not generally exist, owing to the fluctuations in the system as $L \rightarrow \infty$. In the absence of an assumption of the type of (1.9), there may exist sub-domains of $\mathbb{Z}$ where the environment is not propitious for such a bound.

Partial progress may be made using the methods of [21, Sect. 8], but this is too incomplete for inclusion here.

\section{Proof of Theorem 1.4}

Since this proof is very close to that of [21, Thm 2.12], we include only details that are directly relevant to the strengthened claims of the current theorem, namely the removal of the logarithmic term of [21] and the weakened assumption on $\gamma$.

Let $C$ and $\gamma>2 \ln 2$ be as in Theorem 1.2, and choose an integer $K=K(\theta) \geq 2$ such that

$$
C e^{-\gamma K} \leq 1
$$

As in [21],

$$
S\left(\rho_{m}^{L}\right) \leq 2 K, \quad 2 \leq m \leq K,
$$

and we assume henceforth that $m>K$.

Let $\epsilon(r)=C e^{-\gamma(K+r)}$, so that, by (5.1),

$$
\epsilon(r) \leq e^{-\gamma r}, \quad r \geq 0 .
$$

On following the proof of [21, Thm 2.8] up to equation (2.22) there, we find that

$$
\lambda_{j}^{\downarrow}\left(\rho_{m}^{L}\right) \leq \frac{c}{j \xi}, \quad 2^{2 K}<j,
$$

where $\xi=\gamma /(2 \ln 2)>1$ and $c=e^{\gamma(K+1)} /\left(1-e^{-\gamma}\right)$.

Now,

$$
S\left(\rho_{m}^{L}\right)=S_{1}+S_{2}
$$

where

$$
S_{1}=-\sum_{j=1}^{\nu} \lambda_{j}^{\downarrow}\left(\rho_{m}^{L}\right) \log _{2} \lambda_{j}^{\downarrow}\left(\rho_{m}^{L}\right), \quad S_{2}=-\sum_{j=v+1}^{2^{L+1}} \lambda_{j}^{\downarrow}\left(\rho_{m}^{L}\right) \log _{2} \lambda_{j}^{\downarrow}\left(\rho_{m}^{L}\right),
$$

and $v=2^{2(K+2)}$. Since the $\lambda_{j}^{\downarrow}\left(\rho_{m}^{L}\right), 1 \leq j \leq v$, are non-negative with sum $Q$ satisfying $Q \leq 1$, we have

$$
S_{1} \leq \log _{2} v=2(K+2) .
$$


We use (5.4) to bound $S_{2}$ as in [21], to obtain

$$
S_{2} \leq-\sum_{j=v+1}^{\infty} \frac{c}{j^{\xi}} \log _{2}\left(\frac{c}{j^{\xi}}\right) \leq c_{1},
$$

for some $c_{1}=c_{1}(\theta)<\infty$. By (5.5)-(5.6),

$$
S\left(\rho_{m}^{L}\right) \leq 2(K+2)+c_{1}, \quad m \geq K,
$$

which completes the proof.

Open Access This article is distributed under the terms of the Creative Commons Attribution 4.0 International License (http://creativecommons.org/licenses/by/4.0/), which permits unrestricted use, distribution, and reproduction in any medium, provided you give appropriate credit to the original author(s) and the source, provide a link to the Creative Commons license, and indicate if changes were made.

\section{References}

1. Abdul-Rahman, H., Nachtergaele, B., Sims, R., Stolz, G.: Entanglement dynamics of disordered quantum XY chains. Lett. Math. Phys. 106, 649-674 (2016)

2. Aizenman, M., Klein, A., Newman, C.M.: Percolation methods for disordered quantum Ising models. In: Kotecký, R. (ed.) Phase Transitions: Mathematics, Physics, Biology, ..., pp. 129-137. World Scientific, Singapore (1992)

3. Aizenman, M., Nachergaele, B.: Geometric aspects of quantum spin states. Commun. Math. Phys. 164, 17-63 (1994)

4. Arad, I., Kitaev, A., Landau, Z., Vazirani, U.: An area law and sub-exponential algorithm for 1D systems. In: Proceedings of the 4th Innovations in Theoretical Computer Science (ITCS) arXiv.1301.1162 (2013)

5. Arad, I., Landau, Z., Vazirani, U.: Improved one-dimensional area law for frustration-free systems. Phys. Rev. B 85, 195145 (2012)

6. Arad, I., Landau, Z., Vazirani, U., Vidick, T.: Rigorous RG algorithms and area laws for low energy eigenstates in 1D. Commun. Math. Phys. 356, 65-105 (2017)

7. Beaud, V., Sieber, J., Warzel, S.: Bounds on the bipartite entanglement entropy for oscillator systems with or without disorder. J. Phys. A. Math. Theor. 52, 235202 (2019)

8. Beaud, V., Warzel, S.: Bounds on the entanglement entropy of droplet states in the XXZ spin chain. J. Math. Phys. 59, 012109 (2018)

9. Bezuidenhout, C.E., Grimmett, G.R.: Exponential decay for subcritical contact and percolation processes. Ann. Probab. 19, 984-1009 (1991)

10. Bhatia, R.: Matrix Analysis, 2nd edn. Springer, Berlin (1997)

11. Björnberg, J.E.: Vanishing critical magnetization in the quantum Ising model. Commun. Math. Phys. 337, 879-907 (2015)

12. Björnberg, J.E.: The free energy in a class of quantum spin systems and interchange processes. J. Math. Phys. 57, 073303 (2016)

13. Björnberg, J.E., Grimmett, G.R.: The phase transition of the quantum Ising model is sharp. J. Stat. Phys. 136, 231-273 (2009)

14. Brandão, F.G.S.L., Horodecki, M.: An area law for entanglement from exponential decay of correlations. Nat. Phys. 9, 721-726 (2013)

15. Crawford, N., Ioffe, D.: Random current representation for transverse field Ising model. Commun. Math. Phys. 296, 447-474 (2010)

16. Duminil-Copin, H., Raoufi, A., Tassion, V.: Sharp phase transition for the random-cluster and Potts models via decision trees. Ann. Math. 189, 75-99 (2019)

17. Eisert, J., Cramer, M., Plenio, M.B.: Area laws for the entanglement entropy-a review. Rev. Mod. Phys. 82, 277-306 (2010)

18. Goldschmidt, C., Ueltschi, D., Windridge, P.: Quantum Heisenberg Models and Their Probabilistic Representations. Entropy and the Quantum II, Contemporary Mathematics, vol. 552, pp. 177-224. American Mathematical Society, Providence, RI (2011)

19. Grimmett, G.R.: The Random-Cluster Model. Springer, Berlin (2006). http://www.statslab.cam.ac.uk/ $\sim$ grg/books/rcm.html 
20. Grimmett, G.R.: Space-time percolation. In: Sidoravicius, V., Vares, M.E. (eds.) In and Out of Equilibrium 2. Progress in Probability, vol. 60, pp. 305-320. Birkhäuser, Boston (2008)

21. Grimmett, G.R., Osborne, T.J., Scudo, P.F.: Entanglement in the quantum Ising model. J. Stat. Phys. 131, 305-339 (2008)

22. Grimmett, G.R., Stirzaker, D.R.: Probability and Random Processes, 3rd edn. Oxford University Press, Oxford (2001)

23. Nachtergaele, B.: A stochastic geometric approach to quantum spin systems. In: Grimmett, G.R. (ed.) Probability and Phase Transition, pp. 237-246. Kluwer Academic Publishers, Dordrecht (1994)

24. Newman, C.M.: Disordered Ising systems and random cluster representations. In: Grimmett, G.R. (ed.) Probability and Phase Transition, pp. 247-260. Kluwer Academic Publishers, Dordrecht (1994)

25. Pastur, L., Slavin, V.: Area law scaling for the entropy of disordered quasifree fermions. Phys. Rev. Lett. 113, 15404 (2014)

26. Tóth, B.: Improved lower bound on the thermodynamic pressure of the spin $1 / 2$ Heisenberg ferromagnet. Lett. Math. Phys. 28, 75-84 (1993)

Publisher's Note Springer Nature remains neutral with regard to jurisdictional claims in published maps and institutional affiliations. 